\title{
Oral isotretinoin in photoaging: objective histological evidence of efficacy and durability*
}

\author{
Bruna Souza Felix Bravo ${ }^{1,2}$ \\ Ronir Raggio Luiz ${ }^{3}$ \\ Tullia Cuzzi ${ }^{3}$
}

\author{
David Rubem Azulay ${ }^{1,3}$ \\ Carlos Alberto Mandarim-De-Lacerda ${ }^{4}$ \\ Mônica Manela Azulay ${ }^{3}$
}

DOI: http:/ / dx.doi.org/10.1590/abd1806-4841.20153703

\begin{abstract}
BACKGROUND: The off-label use of oral isotretinoin in photoaging is a therapeutic tool that has been used by dermatologists. There are few studies to corroborate its effectiveness and durability.

OвJестіves: To assess, both clinically and histologically, the changes caused by the use of oral isotretinoin in skin photoaging as well as the duration of the effects.

Methods: 20 female patients, aged 45-50 years, with phototypes II-VI, none of whom had experienced menopause, were treated with $20 \mathrm{mg}$ oral isotretinoin, 3 days a week, for 12 weeks. They underwent clinical analysis and skin biopsies in the pre-auricular region, while histologic cuts enabled assessment of the solar elastosis level and morphologic analysis.

RESULTS: Clinically, patients, as well as the researching and the assessor physicians, noticed improvement in skin quality. One patient presented severe solar elastosis, 11 manifested the moderate form, while 8 presented the discreet type. According to histological analysis, $65 \%$ of the patients revealed alteration in the distribution and thickness of the elastic fibers, which can be interpreted as a histological improvement, while $60 \%$ showed an increase in collagen density. We observed an increase in collagen density, from $51.2 \%$ to $57.4 \%,(p=0.004)$. At the end of the 12-week follow-up period, this density decreased to $54.7 \%(p=0.050)$. There was an increase in the density of elastic fibers, from $26.5 \%$ to $31.3 \%$, ( $p=0.02$ ), which had dropped to $27.5 \%$ at the end of the 12 -week follow-up period. CoNCLUSIONS: The study confirmed the role of oral isotretinoin in remodeling the extracellular matrix against photoaging, as well as its durability after 12 weeks, especially when we consider collagen fibers.
\end{abstract}

Keywords: Isotretinoin; Retinoids; Skin aging 


\section{INTRODUCTION}

The skin routinely faces external aggressions such as the wind, humidity, cigarette smoke and (especially) sun exposure. This degeneration process is known as cutaneous extrinsic aging, and the dermatologic effects of its main determining factor, which is widely credited to sun exposure, is known as photoaging. ${ }^{1-3}$

In intrinsic or chronological aging processes, we identify light to moderate sagging, fragility, thinning, and wrinkles. Clinically, we usually differentiate between skin that has aged due to chronic aggression from ultraviolet (UV) radiation and skin that has not suffered due to sun exposure. Alterations are far more visible in UV radiation-exposed skin: more sagging, deep wrinkles, telangectasias, solar melanoses, diffuse hyperpigmentation, dryness, yellowing, leatherlike skin and precancerous lesions. ${ }^{4-6}$

The histological findings from photodamaged skin show epidermal and dermal alterations, especially the hyperplasia of keratinocytes and melanocytes. These cells can display cytological alterations such as an increased nucleus. Solar elastosis is the most prominent histological feature of sun-exposed skin, characterized by the degeneration of elastic fibers in the papillary and reticular dermis, leading to a diffuse homogenization of the conjunctive tissue with inflammatory spots. ${ }^{7}$ Furthermore, it involves the loss of structural support in skin due to deficiencies in collagen types I, III, VII and fibrillin in the papillary dermis. With the loss of such support, dermal blood vessels suffer passive, vascular dilation and an intensification of tortuosity, leading to the appearance of telangiectasias. ${ }^{8}$

Photoaging, however, is treated using procedures which have been widely used and discussed within the dermatologic community. Since Kligman observed a clinical improvement in patients' skin using retinoids for acne in 1986, several other studies have been carried out and this type of medicament has since been regarded as the preferred option for treating cutaneous photodamage. However, side effects such as erythema, scaling and skin burning, as well as the discipline and photoprotection required for this topical treatment, may limit its applicability. Alternatively, dermatologists have been prescribing oral isotretinoin since 1992, upon observing an improvement in the quality of photodamaged skin of patients undergoing regular acne treatment. ${ }^{9}$ Some authors have studied the clinical and histological alterations of this treatment for photoaging. ${ }^{9-12}$ In 2009 , one study demonstrated the effectiveness of this treatment through a histological and morphometric assessment. ${ }^{11}$ The authors then carried out another study to reproduce the previous findings and assess their durability.

\section{METHODS}

\section{Study design}

The study comprises a prospective, clinical study aimed at assessing the effects of oral isotretinoin given to patients to treat cutaneous photoaging thrice a day, three times a week, over 12 weeks, as well as analyzing the effects of treatment 12 weeks after its conclusion. All patients were observed for a period of 24 weeks and underwent a biopsy of the preauricular region with a $5 \mathrm{~mm}$-punch. Further, they underwent photographic documentation, laboratory examinations and clinical assessment, both prior to and 12 weeks after treatment. Histological studies were also carried out to examine the collagen and elastic systems of the 3 skin fragments collected for the study. These studies were complemented by a morphologic study of all samples. Patients were instructed not to wear any sunscreen or dermo-cosmetic products throughout the duration of the study.

\section{Patients}

Twenty healthy female patients aged 45-50 years, with Fitzpatrick phototypes III and IV, clinically diagnosed with moderate (II) to severe (III) photoaging according to the Glogau Scale, of premenopausal status and using safe contraceptive methods, participated in the study. Exclusion criteria comprised women fitting the following profile: pregnant; breastfeeding; of childbearing age but not using a safe contraceptive method; a history of hypercholesterolemia or any ischemic conditions; hepatopathies, esthetic treatments in the 6 months prior to the study such as peeling, laser, dermabrasion, facial lifting or botulinium toxin; dermal or subcutaneous filling; using antiaging creams, weighing under $50 \mathrm{~kg}$ and suffering from any skin illnesses. The study was explained to patients and they were asked about their interest in participating; a consent form was read and signed by those who agreed to take part.

\section{Treatment}

Patients were treated with $20 \mathrm{mg}$ oral isotretinoin twice a day, three times a week, over 12 weeks.

\section{Clinical Assessment}

Patients underwent an anamnesis, focused on the history of their current and previous sun exposure, their current topical and systemic medications, previous dermatologic treatments and existing pathologies. Each patient was clinically examined in order to perform photoaging assessment according to the Glogau Scale, as well as a Fitzpatrick skintype test. Subsequently, each patient was asked to fill in an analog chart analyzing the quality of their skin; researching physicians and assistant physicians 
also completed separate charts for each patient. The charts were filled in both prior to and 12 weeks after treatment; they took into account the following: skin sagging, wrinkles, texture and coloring. Each item was rated from 0 to 10, with 0 being the lowest grade and 10 the highest. At the end of the treatment, patients answered a multiple-choice questionnaire regarding their skin improvement, which featured four options: no improvement $(0-10 \%)$, little improvement (10$40 \%)$, sensible improvement (40-70\%) and high improvement (70-100\%).

\section{Laboratory Analysis}

Prior to treatment and after 12 and 24 weeks, patients provided blood samples for the following tests: human chorionic gonadotropin beta, full blood test, blood glucose testing, alanine transaminase, aspartate aminotransferase, gammaglutamyl transferase, alkaline phosphatase, urea, and creatinine. A test covering $\mathrm{LH}, \mathrm{FSH}$, and testosterone was also asked from patients at the first and last meetings.

\section{Photographic Assessment}

In order to better assess the results of treatment, a trained professional photographed digitally each patient in a studio before treatment commenced, at the end of it and 12 weeks after treatment.

\section{Histopathology}

Skin samples were taken from the left preauricular region through a $5 \mathrm{~mm}$ punch while biopsies were carried out before treatment commenced, at the end of it and 12 weeks later. A $1 \mathrm{~cm}$ margin was observed between each biopsy. The material was preserved in buffered 10\% formaldehyde, following the conventional processing procedures.

Histologic cuts were stained by haematoxylineoslin, picro-sirius and resorcin-fuchsin.

The blades stained by haematoxylin-eoslin obtained through the biopsy carried out prior to treatment enabled us to assess solar elastosis levels as: minor (few basophilic fibers noticed when microscopic magnification was set to medium or high), average (noticeable basophilic material, with fibrillar aspect), or high (with basophilic material mass).

Throughout the morphologic analysis of the collagen and elastic fibers, we took into account, comparatively, in the three sequential biopsies from each patient, the integrity, thickness, density, morphology and orientation of these structures, in addition to the formation of basophilic masses and inflammatory perivasculaer infiltrate.

The histological cuts stained by orcein enabled us to analyze the elastic fibers. Upon analysis, fibers that were stretched, slimmer or oriented were found in less photoaged skin. The worse the actinic damage, the more fragmented and thicker these structures appear. The combined analysis of the histologic cuts stained by haematoxylin-eoslin and of those stained by orcein allowed us to perform a more thorough assessment of solar elastosis.

Through the histologic cuts strained by picrosirus red, we observed a high density of collagen in both the papillary and high reticular dermis. The increase in its thickness, along with a reduction in its spread, was considered an increase in density. Whenever these alterations were not observed, we regarded it as unchanged density.

Later, the blades stained in picro-sirius red and orcein were assessed through morphometric studies to assess the amount of collagen and elastic fibers, comparing them at the two stages of the study. Digital images were obtained from two random blade spots (JPEG format, 36-bit-color, 1280 X1024 pixels) with an LC Evolution camera and an Olympus BX51 microscope. Images were analyzed through the Image Pro Plus software, version 5.0 (Media Cyrnetics, Silver Spring, USA) and then segmented using the same level of semitones to obtain a uniform standard of color and intensity. These images were subsequently transformed into pixels so as to measure the amount of collagen and elastic fibers both before and after treatment.

We selected the pixels corresponding to the collagen fibers (blades stained in picro-sirius red) and elastic fibers (blades stained in orcein). The histogram of the segmentation of the images was assessed and the results from the selected pixels were shown as a percentage of the full image area. Two random spots were photographed from each blade and an average was calculated from the amounts found. This was then defined as the percentage of the elastic or collagen fiber, which was subsequently compared to the posttreatment amounts and those found 12 weeks after treatment.

\section{Statistical Analysis}

Each variable analyzed was evaluated by means of appropriate tables and charts, as well as averages and standard deviation calculations according to the measurement scale for each variable. In the scales assessment, confidence intervals were calculated for the averages. In order to assess pre-/post-treatment and follow-up variation for the variables relating to concentration of collagen and elastic fibers, the variance analysis for repeated data was used, with a posteriori comparisons as per Bonferroni, a significance level of $5 \%$ and SPSS software. 
TABLE 1: Descriptive statistics of elastic and collagen fibers.

\begin{tabular}{|c|c|c|c|c|c|c|}
\hline \multirow{2}{*}{$\begin{array}{l}\text { Descriptive } \\
\text { Statistics }\end{array}$} & \multicolumn{3}{|c|}{ Values \% } & \multicolumn{3}{|c|}{ Differences } \\
\hline & to & t1 & t2 & tl- to & t2- to & t2-t1 \\
\hline \multicolumn{7}{|l|}{ ORCEIN } \\
\hline Average & 26.6 & 31.3 & 27.5 & 4.7 & 0,9 & $-3,8$ \\
\hline Standard deviation & 7.7 & 9.3 & 6.0 & 6.9 & 7,1 & 8,3 \\
\hline \multicolumn{4}{|c|}{ P-value by $t$ test with Bonferroni correction } & 0.020 & 1.000 & 0.165 \\
\hline \multicolumn{7}{|l|}{ PICROSIRIUS } \\
\hline Average & 51.2 & 57.4 & 54.7 & 6.2 & 3,5 & $-2,7$ \\
\hline Standard deviation & 6.0 & 5.1 & 4.5 & 7.3 & 6,0 & 7,2 \\
\hline \multicolumn{4}{|c|}{ P-value by $t$ test with Bonferroni correction } & 0.004 & 0.050 & 0.325 \\
\hline
\end{tabular}

NB: P-value by variance analysis for repeated measures (AN OVA) Orcein $=0.027$; Picrosirius $=0.005$

\section{RESULTS}

The 20 patients completed thestudy showing no signs of significant clinical or laboratory side effects. One patient complained of articular pain in her left elbow that began at the same time as treatment and which stopped with non-hormonal anti-inflammatory medicine, taken over fivedays. Seven patients reported lip dryness and their condition improved after using a lip moisturizer. Another patient mentioned an increase, both in frequency and intensity, of headaches.

All patients wereaged $45-50$ years and the mean age was 47.45 years. With respect to Glogau clinical photoaging classifications, 5 patients (25\%) were type 2, while 15 (75\%) were type 3.

The patients had Fitzpatrick skin phototypes ranging from II-V; 1 (5\%) had phototype II, 8 (40\%) had phototype III, 7 (35\%) had phototype IV, and 4 (20\%) had phototype V. N o patients were menopausal.

We assessed the answers obtained through the questionnaire on the grading of skin quality. According to patients' opinions, the average among initial grades was 5.0, reaching 8.0 at the end of the treatment and 7.2 twel ve weeks after its conclusion.

According to the researching physician, the average grades varied from 6.0 (T0), 8.0 (T1) and 7.3(T2).

The assessing physician graded initial skin quality at 6.4, at the beginning of treatment, 8.4 at the end and 7.5 at the follow-up 12 weeks later.

According to the clinical analysis, taking into account patients' perceptions, 50\% mentioned noticing moderate improvement in the overall quality of their skin (40-70\% improvement), while 50\% reported significant improvement (70-100\% improvement). No patients mentioned mild improvement or absence of improvement (Figures 1, 2, 3).

In the solar elastosis assessment, it was observed that 11 patients presented discreet solar elastosis (55\%), 8 presented average levels (40\%), while $1(5 \%)$ presented high levels of solar elastosis.

The histologic cuts stained by orcein revealed that $65 \%$ of thepatients showed improved morphology and distribution of elastic fibers, which were longer, slimmer, evenly distributed and parallel to the epidermis after treatment. A mong those patients, 76\% had steady levels of improvement in the 12 weeks after treatment. (Figures 4, 5, and 6)

Through the histologic cuts stained by picrosirius red, we assessed the increase in density in the collagen fibers, which was typically parallel to the epidermis. These fibers were thicker and evenly distributed in $60 \%$ of patients. Twelve weeks after treatment, $83 \%$ continued to show an increase in the density of thefibers, even if discreet (Figures 7,8 and 9).

The morphometric analysis was carried out so that the alteration in quantity of elastic and collagen fibers could be assessed. The figures obtained were submitted to the variance analysis with Bonferroni correction.(Table 1)

The histologic cuts stained by orcein were also assessed and a density increase of $26.5-31.3 \%$ was observed in elastic fibers. This $4.7 \%$ variation was statistically relevant $(p=0.02)$, while the standard deviation level was 6.9. At the end of the followup period, this concentration had fallen to $27.5 \%$, maintaining an increase in relation to the period prior to the study, but with no statistical relevance.

An increase in collagen density was observed in the blaces stained by picro-sirius red, rising from $51.2 \%$ to $57.4 \%$, a $6.2 \%$ difference that is statistically relevant $(p=0.004)$, with a standard deviation level of 7.3. Twelve weeks after the end of the treatment, this density decreased to $54.7 \%$, which is higher when compared to the pre-treatment period, with statistically relevant results $(p=0.050)$ and a standard deviation level of 7.2.( Graph 1) 


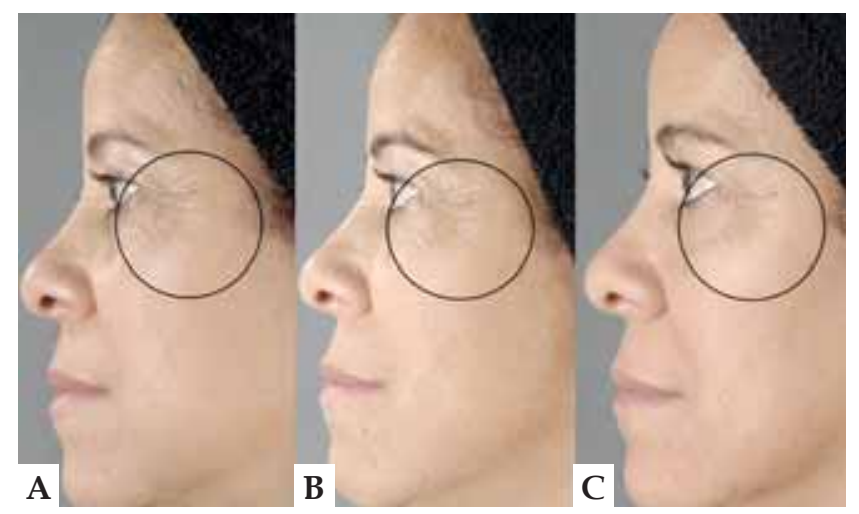

Figure 1: Photograph of a patient's face before (A), after treatment (B) and 12 weeks later (C). There are fewer periorbicular wrinkles and better-defined facial contours

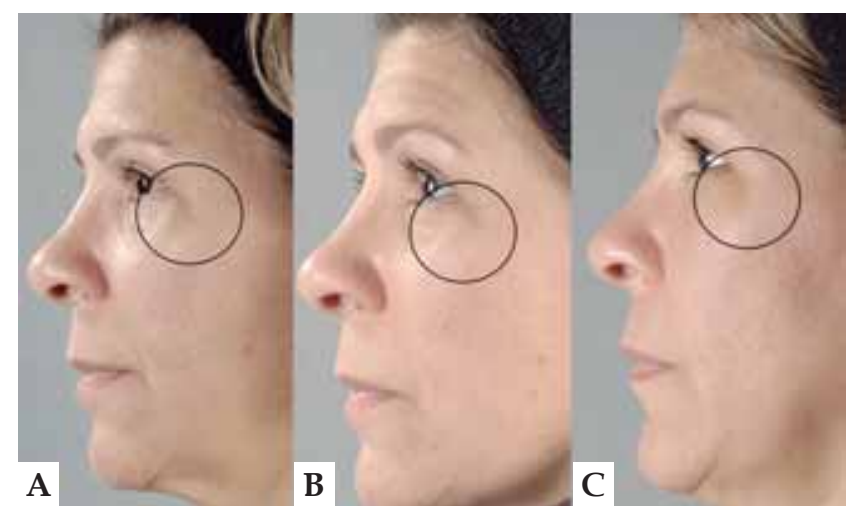

FIGURE 2: Photograph of a patient's face before (A), after treatment (B) and 12 weeks later $(\mathbf{C})$. There are fewer periorbicular wrinkles and better-defined facial contours

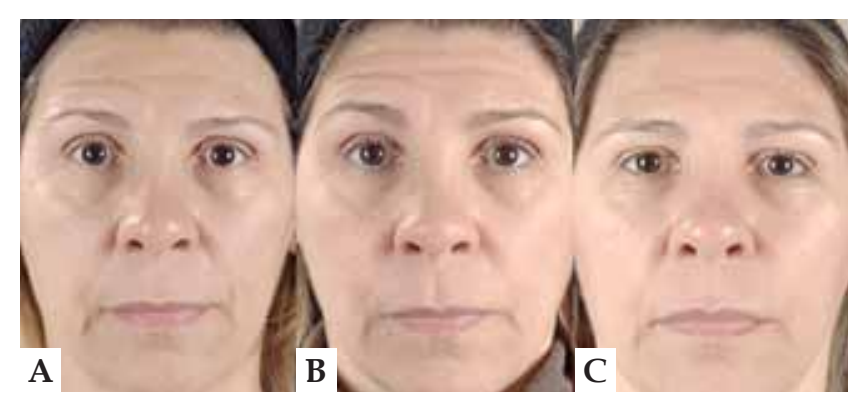

Figure 3: Photograph a patient's face before (A), after treatment (B) and 12 weeks later $(\mathbf{C})$. There is less depth in the nasogenian fold and frontal wrinkles

\section{DISAUSSION}

Drawing on histologic criteria, the results of this study reinforce the clinical view that oral isotretinoin, taken thrice a week in a $20 \mathrm{mg}$ dosage, aids photoaging treatment.

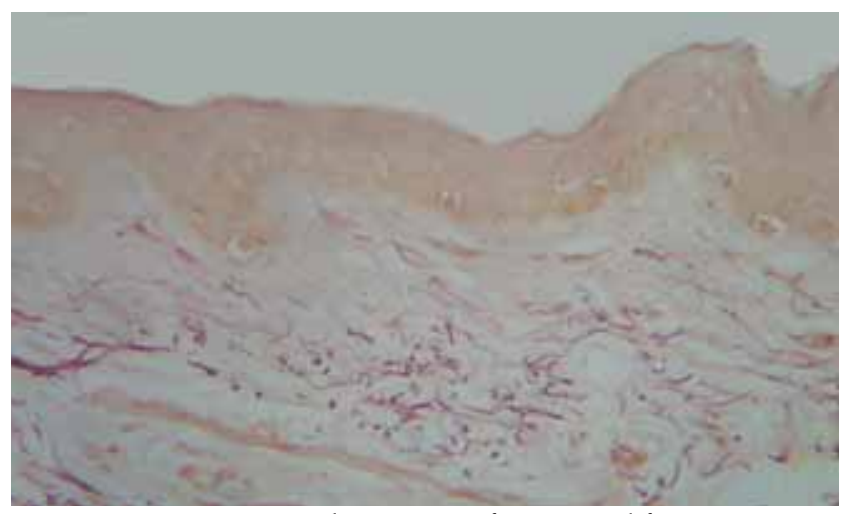

FigURE 4: Pre-treatment biopsy: rarefaction and fragmentation of the elastic fibers in the superficial system and of the elastic fibers in the high reticular dermis. Orcein, obj. X10.

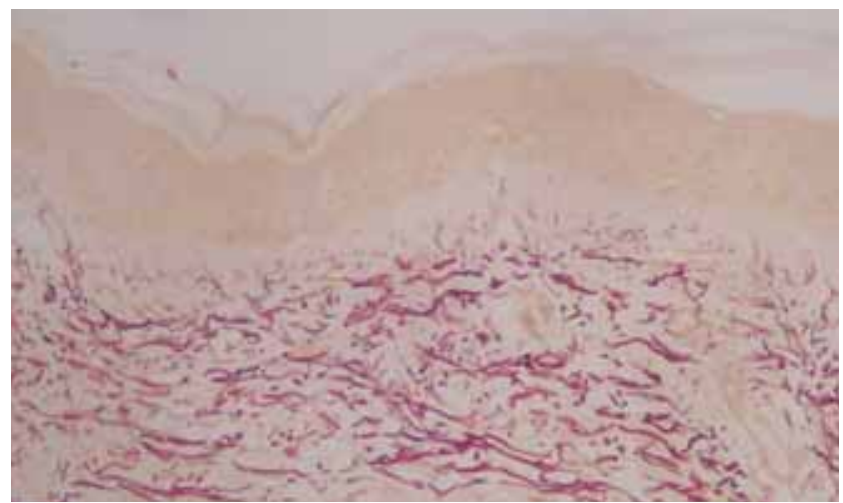

Figure 5: Post-treatment biopsy: Enhancement of the elastic fibers in the superficial system and in the high reticular dermis. Orcein, obj. X10.

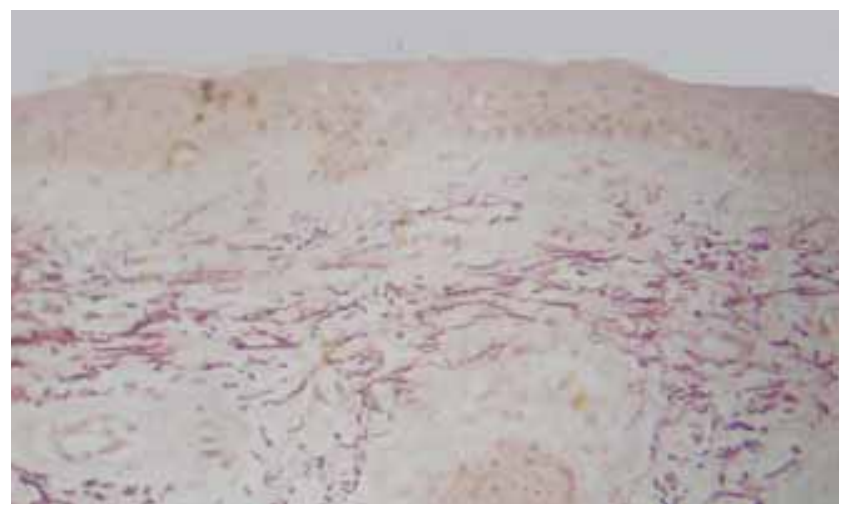

Figure 6: Follow-up biopsy: attenuation of the elastic fibers of the superficial system and maintenance of the characteristics and density of elastic fibers in the reticular high dermis. Orecin, obj X10.

The first study on the use of oral isotretinoin for photoaging, published in 2000 and drawing on subjective analysis, showed a superiority of results from aesthetic, surgical procedures in patients using oral isotretinoin, compared with patients who only 


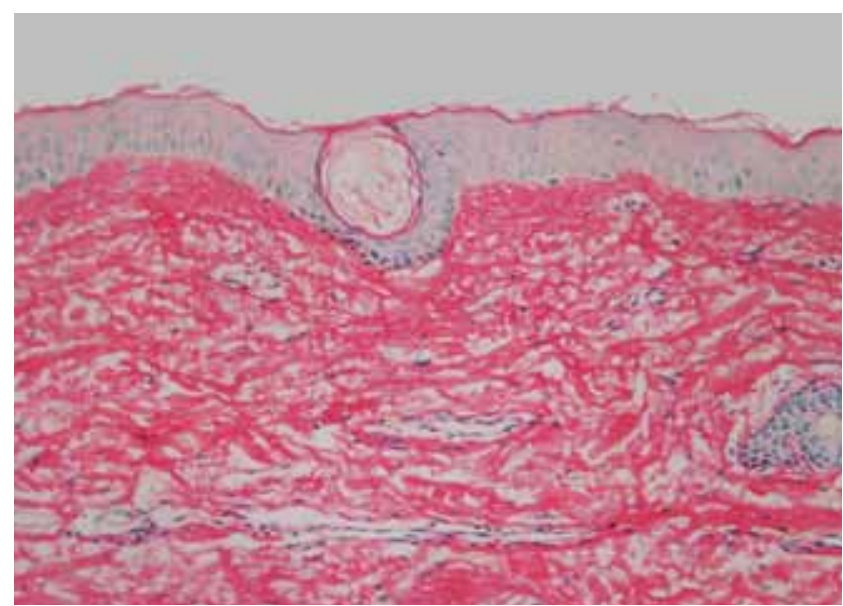

Figure 7: Pre-treatment biopsy: discreet hyalinization of the subepidermal collagen fibers, showing gaps between the collagen fibers in the reticular high dermis. Picro-sirius, obj X 10

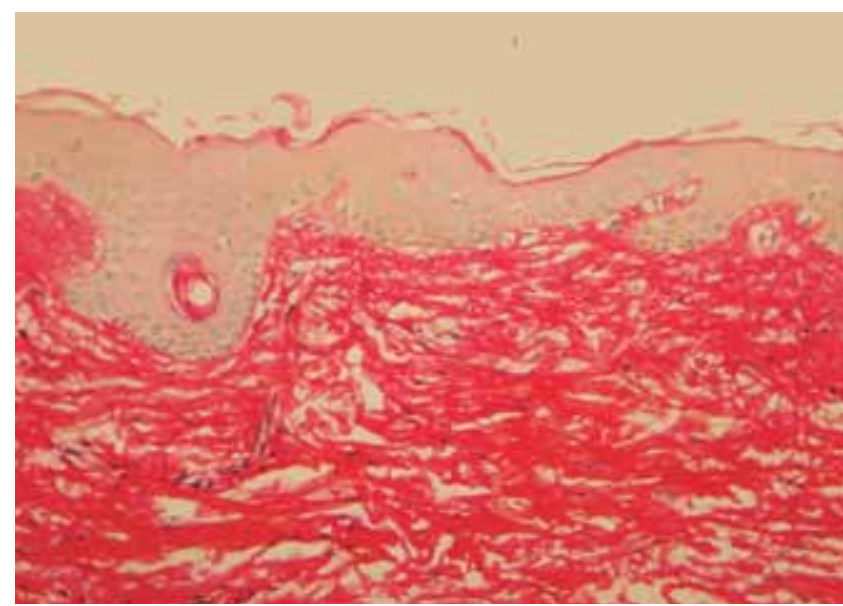

FiguRE 8: Post-treatment biopsy: increase in the density of collagen fibers in the subepidermal dermis. Picro-sirius, obj. X10

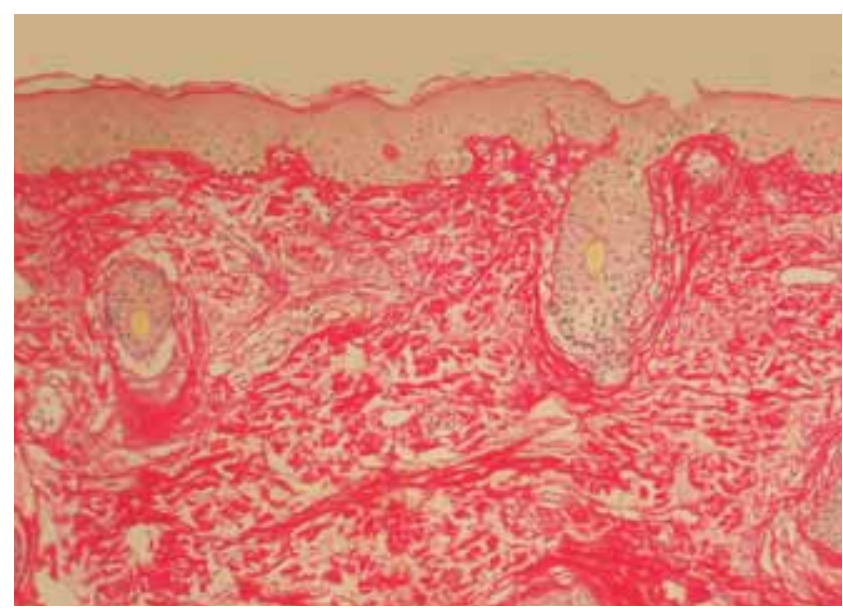

Figure 9: Follow-up biopsy: condensed collagen fibers in the papillary dermis but that are disscociated in the reticular dermis. Picro-sirius, obj X10
Average concentrations via morphometric analysis of elastic (orcein) and collagen (picrosirius) fibers

(pre-, post- and follow-up )

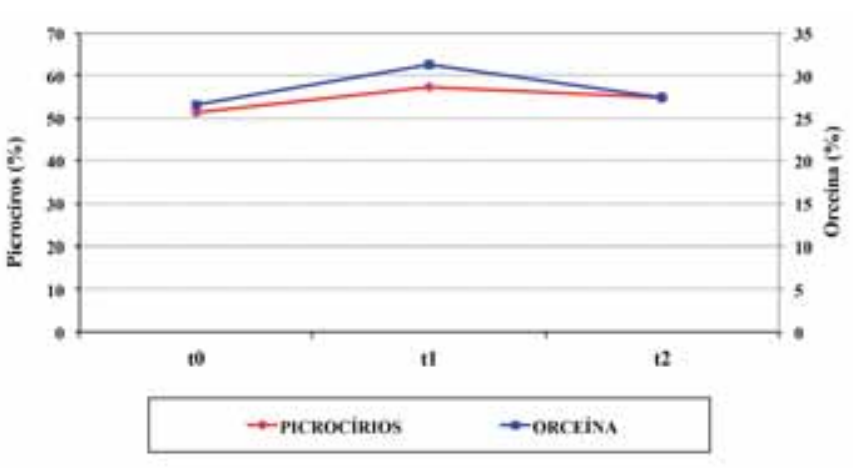

GraPH 1: Morphometric Analysis

underwent procedures via subjective analysis. ${ }^{9}$ Subsequently, three other studies were carried out on the use of oral isotretinoin for photoaging, through histologic analysis. ${ }^{10-12}$ The first study presented a subjective analysis of blades, the second included a morphometric assessment, while the third also included a quantitative, microscopic, digital analysis of the blades, both before and after treatment with oral isotretinoin.

Kalil et al. concluded that there was clinical improvement of solar elastosis, confirmed by histological findings including the correction of dermal papillae and an improvement in collagenic degeneration and cellular tropism.

Rabello-Fonseca described overall improvement in skin quality, a significant increase in the quantity of collagen fibers, as well as a reduction in elastic fibers with a regression of actinic elastosis through morphometric assessment. According to these researchers, there is still room for analysis of the treatment's durability, with the standardization of its dosage, in order to better understand its effects. ${ }^{11}$

Bagatin et al. have described clinical improvement in skin quality, wrinkle depth and elasticity but they did not observe any differences between the group treated with oral isotretinoin, moisturizer and sunscreen, and the group treated only with moisturizer and sunscreen. No meaningful histologic alterations regarding photoaging were found through quantitative microscopic digital analysis, but there was an important reduction in epidermal protein p53 in patients who used oral isotretinoin. ${ }^{12}$

The present study is the fourth offering a histologic assessment within a research line aimed at providing a scientific basis for the already wellestablished dermatological practice of prescribing 
low dosages of oral isotretinoin to treat photoaging. The researchers elected to reduce the age gap between patients so as to have a more homogeneous group. Additionally, they decided to exclude patients who were already menopausal in order to prevent any influence from the considerable reduction in hormones circulating during dermal degeneration. The researchers also carried out an objective analysis of the histological alterations through the morphometrics of the blades, using special staining for elastic fibers and collagen. Despite ethical considerations, the lack of a controlled group using placebo may render the results of the study less accurate.

The main medicine among the arsenal of drugs used against photoaging is topical retinoids, along with alpha hydroxy acids, vitamin $C$ and antioxidants including vitamin $\mathrm{E}$ and others., ${ }^{6,13-20}$ Several non-surgical treatments such as chemical peels, dermabrasion, microneedling, in addition to ablative and non-ablative lasers, are also used against photoaging. ${ }^{21-24}$ However, these treatments have limitations, such as limitations in the area to be treated, lack of discipline from patients due to the need for daily application, and side effects like erythema, itching, burning, not to mention the costs involved and lengthy treatment duration. Oral isotretinoin is a convenient, affordable option for patients, taken in low doses, without significant side effects and, in theory, effective for the skin in general. However, contraceptive care and monthly laboratory tests are essential. ${ }^{25}$

The action mechanism of oral isotretinoin against photoaging still requires further study. There is a theory claiming that this drug inhibits metalloproteinase in the extracellular matrix, leading to a higher production of collagen types I and III. ${ }^{26}$ Furthermore, a more recent study has claimed that it also affects the elastic system, something our own study corroborates. ${ }^{11}$

\section{CONQLUSION}

This study confirmed the effects of oral isotretinoin in remodeling the extracellular matrix against photoaging, as well as its duration 12 weeks after the end of the treatment - especially when taking into account collagen fibers. Further studies are still necessary to better clarify its action mechanism, as well as the subtypes of elastic and collagen fibers involved in this process. 


\section{REFERENCES}

1. Manela-Azulay M. Fotoenvelhecimento. In: Azulay RD, Azulay DR, Azulay-Abulafia L. Azulay Dermatologia. 6. ed. Rio de Janeiro: Guanabara Koogan; 2013. p.893-5.

2. Kligman AM, Grove GL, Hirose R, Leyden JJ. Topical tretinoin for phoytoaged skin. J Am Acad Dermatol. 1986;15:836-59.

3. Matsumura Y, Ananthaswamy HN. Toxic effects of ultraviolet radiation on the skin Toxicol Appl Pharmacol. 2004;195:298-308.

4. Rabe JH, Mamelak AJ, McElgunn PJ, Morison WL, Sauder DN. Photoaging: mechanisms and repair. J Am Acad Dermatol. 2006;55:1-19.

5. Fisher GJ, Kang S, Varani J, Bata-Csorgo Z, Wan Y, Datta S, Voorhees JJ. Mechanisms of Photoaging and Chronological skin aging. Arch Dermatol. 2002;138:1462-70.

6. Kligman LH. Photoaging Manifestations, Prevention, Treatment. Clin Geriatr Med. 1989;5:235-51.

7. Cuzzi-Maya T, Piñeiro-Macieira J. Dermatopatologia: Bases para o diagnóstico morfológico. São Paulo: Editora Roca; 2001. p.74.

8. Singh M, Griffiths CE. The use of retinoids in the treatment of photoaging. Dermatol Ther. 200619:297-305.

9. Hernandez-Perez E, Khawaja HA, Alvarez TY. Oral isotretinoin as part of the treatment of cutaneous aging. Dermatol Surg. 2000;26:649-52.

10. Kalil CL, Fachinello FZ, Lamb FM, Comunello LN. Use of oral isotretinoin in photoaging therapy. Skinmed. 2008;7:10-4.

11. Rabello-Fonseca RM, Azulay DR, Luiz RR, Mandarim-de-Lacerda CA, Cuzzi T, Manela-Azulay M. Oral isotretinoin in photoaging: clinical and histopathological evidence of efficacy of an off-label indication. J Eur Acad Dermatol Venereol. 2009;23:115-23.

12. Bagatin E, Parada MO, Miot HA, Hassun KM, Michalany N, Talarico S. A randomized controlled trial about the use of oral isotretinoin for photoaging. Int $\mathrm{J}$ Dermatol. 2010;49:207-14.

13. Baldwin HE, Nighland M, Kendall C, Mays DA, Grossman R, Newburger J. 40 Years of Topical Tretinoin Use in Review. J Drugs Dermatol. 2013;12:638-42.

14. Cotellessa CPK, Chimenti S. Glycolic acid and its use in dermatology. J Eur Acad Dermatol Venereol. 1995;5:225-17.

15. Green BA, Yu RJ, Van Scott EJ. Clinical and Cosmeceutical uses oh hydroxyacids. Clin Dermatol. 2009;27:495-501.

16. Ditre CM, Griffin TD, Murphy GF, Sueki H, Telegan B, Johnson WC, et al. Effects of Alpha Hydroxy Acids on Photoaged Skin. J Am Acad Dermatol. 1996;34:187-95.

17. Manela-Azulay M, Mandarim-de-Lacerda CA, Perez MA, Filgueira AL, Cuzzi T. Vitamin C. An Bras Dermatol. 2003;78:265-274.

18. Seité S, Bredoux C, Compan D, Zucchi H, Lombard D, Medaisko C, et al. Histological Evaluation of Topically Applied Retinol-Vitamin C Combination. Skin Pharmacol Physiol. 2005;18:81-7.
19. Nachbar F, Korting HC. The role of vitamin E in normal and damaged skin. J Mol Med (Berl). 1995;73:7-17.

20. Yamamoto $\mathrm{Y}$. Role of active oxygen species and antioxidants in photoaging. $\mathrm{J}$ Dermatol Sci. 2001;27:S1-4.

21. Fischer TC, Perosino E, Poli F, Viera MS, Dreno B; Cosmetic Dermatology European Expert Group. Chemical Peels in aesthetic dermatology: an update. J Eur Acad Dermatol Venereol. 2010;24:281-92.

22. Gold MH. Dermabrasion in dermatology. Am J Clin Dermatol. 2003;4:467-71.

23. Doddaballapur S. Microneedling with dermaroller. J Cutan Aesthet Surg. 2009;2:110-1.

24. Papadavid $\mathrm{E}$, Katsambas $\mathrm{A}$. Lasers for facial rejuvenescion : a review. Int $\mathrm{J}$ Dermatol. 2003;42:480-7.

25. Layton AM, Dreno B, Gollnick HP, Zouboulis CC. A review of the European Directive for prescribing systemic isotretinoin for acne vulgaris. J Eur Acad Dermatol Venereol. 2006;20:773-6.

26. Kang S. The mechanism of topical retinoids. Cutis. 2005;75:10-3.

M AILING ADDRESS:

Bruna Souza Felix Bravo

Rua D ona M arina, 143 sala c 11 - Botafogo

22280-020 - Rio de Janeiro - RJ

Brazil

E-mail: brunabravo@globo.com

How to cite this article: Bravo BSF, Azulay DR, Luiz RR, Mandarim-De-Lacerda CA, Cuzzi T, Manela-Azulay M. Oral isotretinoin in photoaging: objective histological evidence of efficacy and durability. An Bras Dermatol. 2015;90(4):478-86. 